(RESEARCH ARTICLE)

\title{
Testing for congruence between the oviposition behaviour of leaf-mining insects and the degree of phylogenetic relatedness among either mining species or hosts species: a case study within the mining moth genus Phyllonorycter (Lepidoptera: Gracillariidae)
}

\author{
Béguinot Jean \\ Société d'Histoire Naturelle-Bourgogne Nature 7 bvd H.P. Schneider 71200 Le Creusot - France.
}

Publication history: Received on 12 October 2019; revised on 08 November 2019; accepted on 10 November 2019

Article DOI: https://doi.org/10.30574/gscarr.2019.1.1.0006

\begin{abstract}
In leaf-mining insects, the oviposition behaviour is especially critical for the welfare of the future brood, the latter being usually doomed to develop entirely within the host-leaf selected for egg-laying by the ovipositing females. As, moreover, oviposition behaviour in leaf miners depends upon the taxonomic identities of both the mining-insect and the host, one can thus speculate that the patterns of oviposition behaviour of different leaf-mining species could be more or less congruent with either the degree of phylogenetic relatedness of the leaf-mining species themselves or the degree of phylogenetic relatedness of their respective host-species. Here, I test successively these two hypotheses - the "miners phylogenetic relatedness" hypothesis and the "hosts phylogenetic relatedness" hypothesis - by addressing a system insect-plant involving four mining moth species (all four belonging to the genus Phyllonorycter) and the three corresponding host-tree species, all included within the family Betulaceae. It turns out that, for this system at least, neither of the two previous hypotheses is actually supported. Possible reasons for this double rejection are discussed accordingly.
\end{abstract}

Keywords: Egg-laying behaviour; Selectivity; Host acceptance

\section{Introduction}

Quantitative aspects of the oviposition behaviour in leaf-mining insects - the severity of ovipositing mothers in selecting host-leaves accepted for subsequent egg-laying (the "leaf-acceptance ratio") and the average clutch-size - both play significant role in determining the future successful development of offspring and, thereby, are intended to have direct influence on the intensity of the resulting mining attack [1, 2]. These behavioural traits are reactions of ovipositing females following leaf probing and, accordingly, these behavioural traits should depend upon both:

(i) the foliage quality of the host species, as perceived "in the eyes of the mining-species" [3] (i.e. a host-related parameter) and

(ii) the corresponding level of requirement of mothers themselves, as regards the minimal quality of the host-leaf judged by mothers as being acceptable for subsequent egg-laying (i.e. an insect-related parameter).

Accordingly, any significant behavioural differences that might be disclosed between different mining-species within a same mining genus attacking different hosts species, would likely result from difference regarding either the host and/or the mining species.

\footnotetext{
${ }^{*}$ Corresponding author

E-mail address: jean-beguinot@orange.fr
} 
Now, the shared responsibilities of the insect and of the host-plant in this respect may be tracked by seeking for the existence of a possible congruence between:

(i) the respective oviposition behaviours of different mining species and

(ii) the pattern of phylogenetic relatedness either among these mining species themselves (the "miners phylogenetic relatedness" hypothesis) or among the corresponding hosts species (the "hosts phylogenetic relatedness" hypothesis).

Hereafter, I successively address and test these two hypotheses. The system \{insects-hosts\} considered for this study involves four moth species, all belonging to the genus Phyllonorycter, mining the leaves of one or the other of three host species representative of the family Betulaceae (Alnus glutinosa, Carpinus betulus, Corylus avellana). This system was chosen because we dispose of recent phylogenies published for Phyllonorycter [4] and for Betulaceae [5].

\section{Material and methods}

As already mentioned, egg-laying behaviour of leaf-mining mothers may be characterised by two major quantitative parameters: the leaf-acceptance ratio ' $\alpha$ ' that reflect the degree of mothers' selectivity for leaf quality prior to oviposition and the average clutch-size ' $n_{c}$ ' (the number of eggs deposited as a single bout on a previously accepted host-leaf). These behavioural parameters are, yet, very difficult to directly observe reliably under field conditions. It is possible, however, to trace back to these parameters by subsequently considering the resulting pattern of mines distribution among hostleaves. Accordingly, a specific approach, the so-called "Melba" procedure, has been developed in this purpose [6 - 12]. This procedure was implemented here and successively applied to 19 stands supporting mining attacks of:

- Phyllonorycter coryli (Nicelli, 1851) (7 stands) and Ph. nicellii (Stainton, 1851) (5 stands) on Corylus avellana L.,

- Ph. esperella (Goeze, 1783) (6 stands) on Carpinus betulus L. and

- Ph. rajella (Linnaeus, 1758) (1 stand) on Alnus glutinosa (L.) Gaertner.

Running the "Melba" procedure provides estimations of the leaf-acceptance ratio ' $\alpha$ ' and the average clutch-size ' $\mathrm{nc}$ ' for each of the sampled stands. Pooled results are presented in figure1.

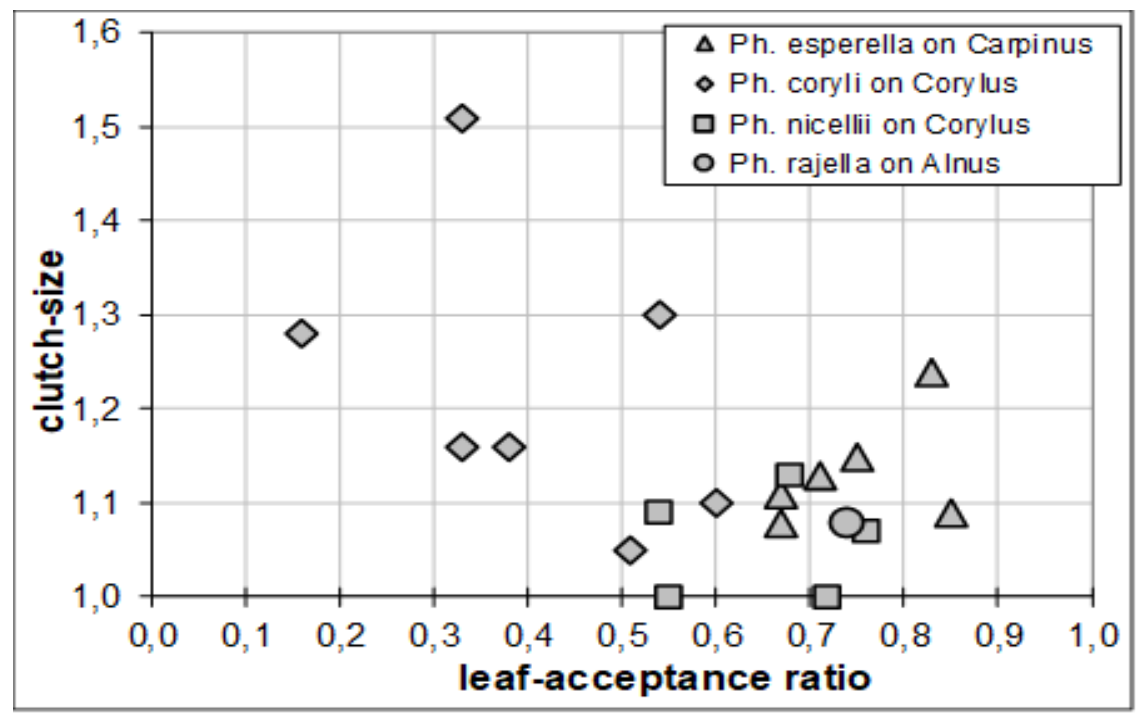

Figure 1 The distribution of (i) the leaf-acceptance ratio $\alpha$ and (ii) the average clutch-size $\mathrm{n}_{\mathrm{c}}$ for Phyllonorycter coryli (7 stands) and Ph. nicellii (5 stands) on Corylus avellana; for Ph. esperella (6 stands) on Carpinus betulus and for Ph. rajella (1 stand) on Alnus glutinosa. 


\section{Results and discussion}

\subsection{Testing for the congruence between insect behaviour and the corresponding host-tree phylogenetic relatedness (within Betulaceae)}

The three genus Alnus, Carpinus and Corylus all belong to the family Betulaceae, but Alnus on the one hand and Carpinus and Corylus on the other hand are members of well differentiated tribes within Betulaceae: Betuloideae for Alnus, Coryloideae for Carpinus and Corylus [5].

The pattern of combined distribution of the two oviposition behaviour parameters - the leaf-acceptance ratio ' $\alpha$ ' and the average clutch-size ' $n c$ ' - (figure 1 ) hardly mirrors the respective degrees of phylogenetic relatedness between the corresponding host-tree species (figure 2). Thus, the distribution of combined values $\left[\alpha, \mathrm{n}_{c}\right]$ for $P h$. nicellii is closer to the corresponding distributions for Ph. esperella and Ph. rajella than it is to the distribution for Ph. coryli. And this, although Ph. nicellii and Ph. coryli share the same host-species, Corylus avellana, while Ph. nicellii, Ph. esperella and $P h$. rajella are mining different host-species, including Alnus, belonging to a different tribe. Therefore, no congruence occurs between the respective patterns of behavioural parameters of each of the four moth species and the pattern of phylogenetic relatedness among their respective host-species. Thus, here, the "hosts phylogenetic relatedness" hypothesis is not supported.

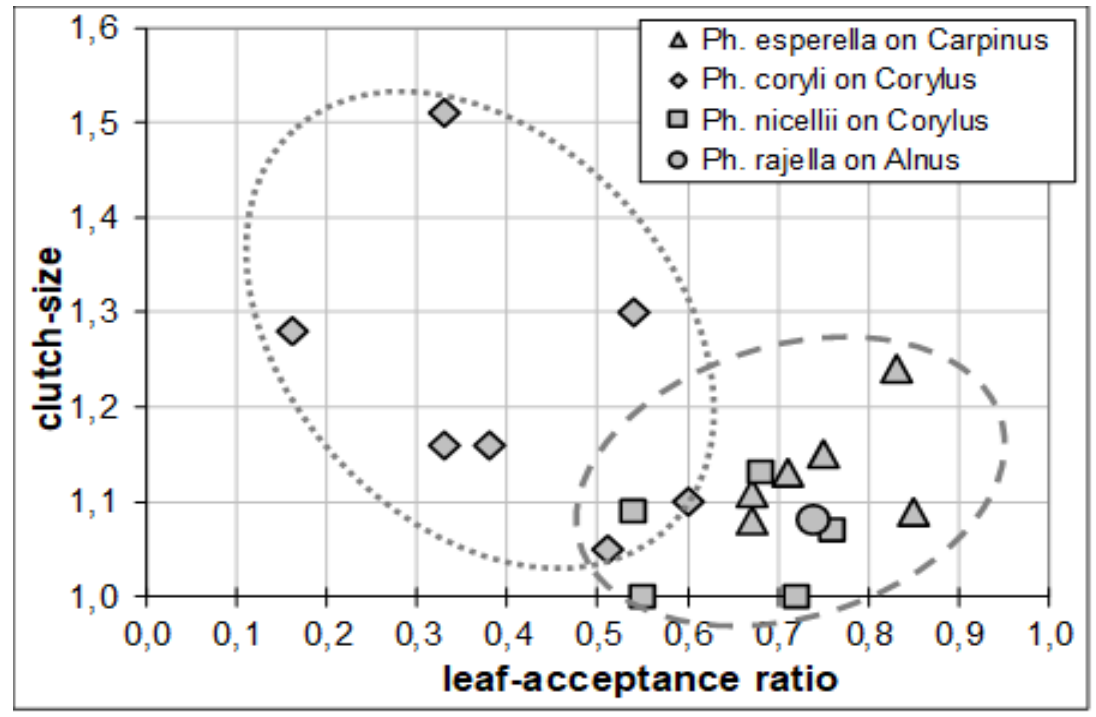

Figure 2 Non-congruence between the pattern of oviposition behaviour (the leaf-acceptance $\alpha$ and clutch-size nc) and the phylogenetic relatedness of the respective host species [5]. Comments in text.

In figure 2, the dashed contour delimits similar behavioural parameters for three moths, Ph. nicellii, Ph. esperella and Ph. rajella, although each of them is mining different hosts - Corylus avellana, Carpinus betulus, Alnus glutinosa respectively. Moreover, these three host-species belong to different tribes in Betulaceae. On the contrary, Ph. coryli (dotted contour) and Ph. nicelii (dashed contour) significantly differ in their respective behavioural parameters (Mann \& Whitney test, $\mathrm{p}<0.05$ regarding both the differences on leaf-acceptance $\alpha$ and clutch-size $\mathrm{n}_{c}$ ) although they share the same host-species, Corylus avellana. Accordingly, the "hosts phylogenetic relatedness" hypothesis is not supported.

\subsection{Testing for the congruence between insects behaviours and their own phylogenetic relatedness pattern within the genus Phyllonorycter}

According to [4], closely related Phyllonorycter species generally feed on closely related hosts. Yet, I test whether the same holds true for egg-laying behaviour: figure 3.

First, among the four mining species involved, Ph. coryli and Ph. esperella are closely related [4] and, indeed, share the same (unusual) type of mining habit: upper-surface tentiforme mines. The two other moth species, Ph. nicellii and $P h$. rajella - although being distantly related [4] - yet induce both the (common type) lower-surface tentiforme mines. 
Second, the patterns of oviposition behaviours of the four mining moths, in terms of combined values of $\alpha$ and $\mathrm{n}_{\mathrm{c}}$, do not show any appreciable congruence with the degrees of phylogenetic relatedness between them (Figure 3). Thus, here, the "miners phylogenetic relatedness" hypothesis is not supported.

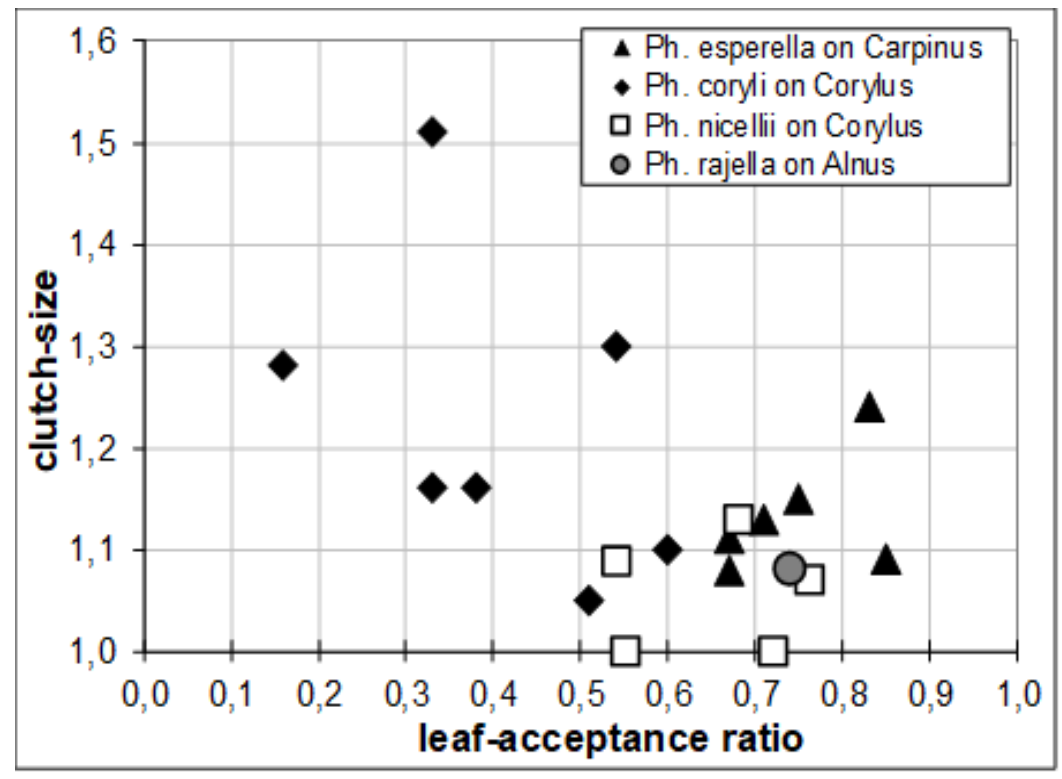

Figure 3 The distribution of combined values of $\alpha \& \mathrm{n}_{\mathrm{c}}$ of the four mining moths tested against the phylogenetic relatedness between them. Comments in text.

In figure 3 and according to [4], the degree of similarity of colours of figures is intended to mirror the degree of phylogenetic relatedness between mining-moths species: black for both the closely related Ph. coryli and Ph. esperella; grey for the distant Ph. rajella and white for the still more distant Ph. nicellii. Instead of being segregated apart, the distributions of values of $\alpha \& \mathrm{n}_{\mathrm{c}}$ for the distantly related species Ph. esperella, Ph. rajella and Ph. nicellii are included within a same scatter, while the distribution of $\alpha$ and $\mathrm{n}_{\mathrm{c}}$ for Ph. coryli is segregated apart, in spite of its close relatedness to Ph. esperella. Accordingly, the "miners phylogenetic relatedness" hypothesis is not supported.

\subsection{Discussion of results}

"Pre-hatching maternal-care behaviour" in leaf-miners (involving both (i) the host-leaf acceptance ratio for subsequent egg deposit and (ii) the size of egg-clutch), is likely intended to (i) selecting the best resource insuring the efficient development of the future brood and (ii) preventing the risk of scramble competition between conspecific larvae within a same host leaf, respectively [1-2]. Accordingly, this pre-hatching maternal-care will arguably contribute to the optimal development of offspring and, thus, deserve attention, in particular regarding the causes of variations in oviposition behaviour among mining species.

As such, these behaviours should account for both the taxonomic identity of the moth and the taxonomic identity of the host. Which may suggest, in turn, that some congruence could exist between the patterns of behavioural parameters of the egg-laying mothers and the patterns of phylogenetic relatedness between either the mining moth-species or the corresponding host-species [13]. What we called respectively the "miners phylogenetic relatedness" hypothesis and the "hosts phylogenetic relatedness" hypothesis.

Within the system considered here, including four Phyllonorycter moths, each of them mining one among three hostspecies, all belonging to the family Betulaceae, no congruence appears between the pattern of differentiated oviposition behaviours of the four mining-moth species and the patterns of phylogenetic relatedness among the mining-moths as well as among their respective host-trees. So that neither the "miners phylogenetic relatedness" hypothesis nor the "hosts phylogenetic relatedness" hypothesis are supported, at least for the limited system investigated here.

This, finally, might not be so surprising. Indeed, a congruence between the patterns of differentiated moth behaviours and the pattern of phylogenetic relatedness of host-trees would likely be expected only if none of the four moths had had enough micro-evolutionary time to undergo substantial adaptative evolution, in order to cope at best with their respective preferred hosts. In other words, such congruence might have been expected only if the four moth species had not already undergone substantial evolutive differentiation allowing them to adapt to their respective hosts 
independently of the degree of relatedness of the latter: see reference [12] and, here, Ph. coryli and Ph. nicelli having adapted to mining a same host, Corylus avellana, in spite of their own distant relatedness: figure 3). But, on the contrary, it is more likely that each of these mining species is already well advanced in its own micro-evolutive history. So that, difference in oviposition behaviours among the four mining species have less reason to still reflect, at present time, the degree of difference between the phylogenetic traits of their respective hosts.

The same type of argument might explain the lack of congruence between the pattern of oviposition behaviour and the pattern of phylogenetic relatedness among the mining-moths themselves: the micro-evolution of each moth species is intended to adapt each species to its particular host and, thus, tends to reduce, more or less, the differences between the degrees of selectivity expected between moths.

\section{Conclusion}

No congruence was disclosed between the main descriptors of oviposition behaviour and the degrees of phylogenetic relatedness among the four studied mining species within the mining moth genus Phyllonorycter. No congruence either was detected between the main descriptors of oviposition behaviour and the degrees of phylogenetic relatedness among the three host-trees involved in the study. Accordingly, neither the "miners' phylogenetic relatedness" hypothesis nor the "hosts phylogenetic relatedness" hypothesis are supported in the system insect-host plant considered here. Yet, although some rational has been suggested to explain this double rejection (i.e. some close and specific miner-host adaptations seem likely to have evolved independently in each couple insect-host), it would remain useful to further extend the investigation to a series of other systems of leaf-miners and their associated host-species. Indeed, tentatively generalizing from one case study only - would this generalization might seem rather logical - is still somewhat adventurous.

\section{Compliance with ethical standards}

\section{Acknowledgments}

Author acknowledges comments from the Editor on the original version of the manuscript.

\section{Disclosure of conflict of interest}

The author declares no conflict of interest regarding this study.

\section{References}

[1] Paukku S and Kotiaho JS. (2008). Female Oviposition Decisions and Their Impact on Progeny Life-History Traits. Journal of Insect Behaviour, 21(6), 505-520.

[2] Bonebrake T, Boggs CL, McNally JH, Ranganathan J and Ehrlich PR. (2010). Oviposition behavior and offspring performance in herbivorous insects: Consequences of climatic and habitat heterogeneity. Oikos, 119(6), 927-934.

[3] Gripenberg S, Salminen JP and Roslin T. (2007). A tree in the eyes of a moth - temporal variation in oak leaf quality and leaf-miner performance. Oikos, 116, 592-600.

[4] Lopez-Vaamonde C, Godfray HCJ and Cook JM. (2003). Evolutionary dynamics of host-plant use in a genus of leaf-mining moths. Evolution, 57(8), 1804-1821.

[5] Chen ZD, Manchester SR and Sun HY. (1999). Phylogeny and evolution of the Betulaceae as inferred from DNA sequences, morphomogy and paleobotany. American Journal of Botany, 86(8), 1168-1181.

[6] Béguinot J. (2011). Comparison of the severity of selection among beech leaves prior to egg-laying between a leaf-mining and two gall-inducing insects. European Journal of Entomology, 108, 71-78.

[7] Béguinot J. (2012a). Quantifying the gap between the actual incidence of leaf-mining or leaf-galling insects and the proportion of host-leaves potentially acceptable by them. Annales de la Société Entomologique de France, 48 (1-2), 216-224.

[8] Béguinot J. (2012b). Would mothers relax their degree of selectivity for supports suitable for egg-laying when the local density of conspecific females increases? A case study with three common lepidopteran leaf-miners. ISRN Zoology. 
[9] Béguinot J. (2013). L'invasion éruptive de la chenille mineuse des feuilles de Marronnier, Cameraria ohridella (Lepidoptera: Gracillariidae). Caractérisation quantitative des facteurs comportementaux et environnementaux responsables. Revue scientifique Bourgogne Nature 18, 87-91.

[10] Béguinot J. (2014). Would clutch-size decrease with higher local-density of mothers and/or lower quality of the host, in leaf-mining moths? A case study with three common lepidopteran leaf-miners. Revue d'écologie (la Terre et la Vie), 69(3/4), 258-266.

[11] Béguinot J. (2015). Quantitative aspects of egg-laying behaviour contribute to the eruptive success of Cameraria ohridella parasiting horse-chestnuts. Journal of Biology and Nature, 2(3), 106-110.

[12] Béguinot J. (2018). On some quantitative traits of maternal care prior to egg-laying in mining moths. Revue Scientifique Bourgogne-Franche-Comté Nature, 28, 135-143.

[13] Mitter C, Farrell BD and Futuyma DJ. (1991). Phylogenetic studies of insect-plant interactions: insights into the genesis of diversity. Trends in Ecology \& Evolution, 6(9), 290-293.

\section{How to cite this article}

Béguinot J. (2019). Testing for congruence between the oviposition behaviour of leaf-mining insects and the degree of phylogenetic relatedness among either mining species or hosts species: a case study within the mining moth genus Phyllonorycter (Lepidoptera: Gracillariidae). GSC Advanced Research and Reviews, 1(1), 04-09. 